\title{
The gamma extended Weibull family of distributions
}

\author{
Abraão D. C. Nascimento ${ }^{1 *}$, Marcelo Bourguignon ${ }^{1}$, Luz M. Zea ${ }^{1}$, Manoel Santos-Neto ${ }^{2}$, Rodrigo B. Silva ${ }^{1}$ and \\ Gauss M. Cordeiro ${ }^{1}$ \\ ${ }^{1}$ Departamento de Estatística, Universidade Federal de Pernambuco (UFPE), \\ Cidade Universitária, s/n, 50740-540, Recife, PE, Brasil \\ 2 Departamento de Estatística, Universidade Federal de Campina Grande (UFCG), \\ 58109-970, Campina Grande, PB, Brasil
}

Received 20 April 2013

Accepted 26 June 2013

\begin{abstract}
We introduce a new family of distributions called the gamma extended Weibull family. The proposed family includes several well-known models as special cases and defines at least seventeen new special models. Structural properties of this family are studied. Additionally, the maximum likelihood method for estimating the model parameters is discussed. An application to real data illustrates the usefulness of the new family. The results provide evidence that the proposed family outperforms other classes of lifetime models.

Keywords: Extended Weibull distributions; Hazard rate function; Maximum likelihood estimation; Moments; Stacy's generalized gamma distribution.
\end{abstract}

\section{Introduction}

Recently, Zografos and Balakrishnan [24] introduced and studied a broad family of univariate distributions through a particular case of Stacy's generalized gamma distribution, in the same way as Jones's family is defined following the beta distribution. This new family stems from the general class: if $\mathrm{G}$ denotes the baseline cumulative distribution function (cdf) of a random variable, then a generalized class of distributions can be defined by

$$
F(x ; \delta)=\gamma\{\delta,-\log [1-G(x)]\},
$$

where $x \in \mathscr{X} \subseteq \mathbb{R}, \delta>0, \gamma(\delta, z)=\Gamma(\delta)^{-1} \int_{0}^{z} t^{\delta-1} \mathrm{e}^{-\mathrm{t}} \mathrm{dt}$ denotes the incomplete gamma function and $\Gamma(\cdot)$ is the gamma function. This family of distributions has probability density function (pdf) given by

$$
f(x ; \delta)=\frac{1}{\Gamma(\delta)}\{-\log [1-G(x)]\}^{\delta-1} g(x)
$$

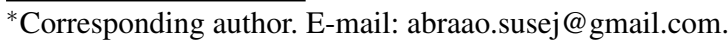


Moreover, the class of extended Weibull ( $\mathscr{E} \mathscr{W})$ distributions, as proposed by Gurvich et al. [8], has achieved a prominent position in new probability models. Its cdf is

$$
G(x ; \alpha, \boldsymbol{\xi})=1-\exp [-\alpha H(x ; \boldsymbol{\xi})]
$$

where $x \in \mathscr{D} \subseteq \mathbb{R}_{+}, \alpha>0$ and $H(x ; \boldsymbol{\xi})$ is a non-negative monotonically increasing function which depends on the parameter vector $\boldsymbol{\xi}$. The corresponding pdf is given by

$$
g(x ; \alpha, \boldsymbol{\xi})=\alpha \exp [-\alpha H(x ; \boldsymbol{\xi})] h(x ; \boldsymbol{\xi}),
$$

where $h(x ; \boldsymbol{\xi})$ is the derivative of $H(x ; \boldsymbol{\xi})$.

Note that different functions $H(x ; \boldsymbol{\xi})$ in equation (1.3) yield important statistical models such as: $H(x ; \boldsymbol{\xi})=x$ gives the exponential distribution; $H(x ; \boldsymbol{\xi})=x^{2}$ leads to the Rayleigh distribution; $H(x ; \boldsymbol{\xi})=\log (x / k)$ leads to the Pareto distribution and $H(x ; \boldsymbol{\xi})=\beta^{-1}[\exp (\beta x)-1]$ gives the Gompertz distribution. Table 1 displays the functions $H(\cdot ; \cdot)$ and $h(\cdot ; \cdot)$ and the corresponding parameter vectors for special distributions.

In this paper, we derive a new family of distributions by compounding the classes of gamma and $\mathscr{E} \mathscr{W}$ distributions. The compounding procedure follows by taking the $\mathscr{E} \mathscr{W}$ family of distributions as the baseline distribution in (1.1). The gamma extended Weibull $(\mathscr{G} \mathscr{E} \mathscr{W})$ family of distributions contains as special models the modified Weibull, Pareto and Gompertz distributions, among those listed in Table1.

The paper is organized as follows. In Section 2, we define the $\mathscr{G} \mathscr{E} \mathscr{W}$ class of distributions and obtain useful expansions for its cumulative and density functions. Some mathematical properties are derived and discussed in Sections 3 6r quantile function, order statistics, generating function, incomplete moments and mean deviations. Additionally, some information theory measures for the proposed family are derived. Formulas for the Rényi and Shannon entropies are presented in Section 7 and 8 , respectively. In Section 9, we present expressions for the cross entropy and Kullback-Leibler divergence. The maximum likelihood method and the observed information matrix are investigated in Section 10. Some special cases are studied in some detail in Section 11. An application to a real data set is performed in Section 12 in order to illustrate the flexibility and potentiality of the new family. Finally, main conclusions are addressed in Section 13 .

\section{The $\mathscr{G} \mathscr{E} \mathscr{W}$ family of distributions}

Taking the $\mathscr{E} \mathscr{W}$ family of distributions as the baseline model in equation 1.1, we have

$$
F(x ; \delta, \alpha, \boldsymbol{\xi})=\gamma[\delta, \alpha H(x ; \boldsymbol{\xi})],
$$

where $x \in \mathscr{D}, \alpha>0$ and $\delta>0$. The corresponding pdf has a very simple form

$$
f(x ; \boldsymbol{\delta}, \alpha, \boldsymbol{\xi})=\frac{\alpha^{\delta}}{\Gamma(\boldsymbol{\delta})} h(x ; \boldsymbol{\xi}) H(x ; \boldsymbol{\xi})^{\delta-1} \exp [-\alpha H(x ; \boldsymbol{\xi})]
$$

where $H(x ; \boldsymbol{\xi})$ corresponds to a special distribution listed in Table 1 with cdf given in 1.3 . 
Table 1. Special distributions and corresponding $H(x ; \boldsymbol{\xi})$ and $h(x ; \boldsymbol{\xi})$ functions

\begin{tabular}{|c|c|c|c|c|}
\hline Distribution & $H(x ; \boldsymbol{\xi})$ & $h(x ; \boldsymbol{\xi})$ & $\alpha$ & $\boldsymbol{\xi}$ \\
\hline Exponential $(x \geq 0)[9]$ & $x$ & 1 & $\alpha$ & $\emptyset$ \\
\hline Pareto $(x \geq k)[9]$ & $\log (x / k)$ & $1 / x$ & $\alpha$ & $k$ \\
\hline Rayleigh $(x \geq 0) \mid 18$ & $x^{2}$ & $2 x$ & $\alpha$ & $\emptyset$ \\
\hline Weibull $(x \geq 0)$ & $x^{\gamma}$ & $\gamma x^{\gamma-1}$ & $\alpha$ & $\gamma$ \\
\hline Modified Weibull $(x \geq 0)[11]$ & $x^{\gamma} \exp (\lambda x)$ & $x^{\gamma-1} \exp (\lambda x)(\gamma+\lambda x)$ & $\alpha$ & {$[\gamma, \lambda]$} \\
\hline Weibull extension $(x \geq 0)[23]$ & $\lambda\left[\exp (x / \lambda)^{\beta}-1\right]$ & $\beta \exp (x / \lambda)^{\beta}(x / \lambda)^{\beta-1}$ & $\alpha$ & {$[\gamma, \lambda, \beta]$} \\
\hline Log-Weibull $(-\infty<x<\infty)[21]$ & $\exp [(x-\mu) / \sigma]$ & $(1 / \sigma) \exp [(x-\mu) / \sigma]$ & 1 & {$[\mu, \sigma]$} \\
\hline Phani $(0<\mu<x<\sigma<\infty) \mid \overline{17}$ & {$[(x-\mu) /(\sigma-x)]^{\beta}$} & $\begin{array}{l}\beta[(x-\mu) /(\sigma-x)]^{\beta-1} \\
\times\left[(\sigma-\mu) /(\sigma-t)^{2}\right]\end{array}$ & $\alpha$ & {$[\mu, \sigma, \beta]$} \\
\hline Weibull Kies $(0<\mu<x<\sigma<\infty)[10]$ & $(x-\mu)^{\beta_{1}} /(\sigma-x)^{\beta_{2}}$ & $\begin{array}{l}(x-\mu)^{\beta_{1}-1}(\sigma-x)^{-\beta_{2}-1} \\
\times\left[\beta_{1}(\sigma-x)+\beta_{2}(x-\mu)\right]\end{array}$ & $\alpha$ & {$\left[\mu, \sigma, \beta_{1}, \beta_{2}\right]$} \\
\hline Additive Weibull $(x \geq 0)$ 22 & $\left(x / \beta_{1}\right)^{\alpha_{1}}+\left(x / \beta_{2}\right)^{\alpha_{2}}$ & $\begin{array}{l}\left(\alpha_{1} / \beta_{1}\right)\left(x / \beta_{1}\right)^{\alpha_{1}-1} \\
+\left(\alpha_{2} / \beta_{2}\right)\left(x / \beta_{2}\right)^{\alpha_{2}-1}\end{array}$ & 1 & {$\left[\alpha_{1}, \alpha_{2}, \beta_{1}, \beta_{2}\right]$} \\
\hline Traditional Weibull $(x \geq 0)$ & $x^{b}\left[\exp \left(c x^{d}-1\right)\right]$ & $\begin{array}{l}b x^{b-1}\left[\exp \left(c x^{d}\right)-1\right] \\
+c d x^{b+d-1} \exp \left(c x^{d}\right)\end{array}$ & $\alpha$ & {$[b, c, d]$} \\
\hline Gen. power Weibull $(x \geq 0) \mid 15$ & {$\left[1+(x / \beta)^{\alpha_{1}}\right]^{\theta}-1$} & $\left(\theta \alpha_{1} / \beta\right)\left[1+(x / \beta)^{\alpha_{1}}\right]^{\theta-1}(x / \beta)^{\alpha_{1}}$ & 1 & {$\left[\alpha_{1}, \beta, \theta\right]$} \\
\hline Flexible Weibull extension $(x \geq 0) \mid 1]$ & $\exp \left(\alpha_{1} x-\beta / x\right)$ & $\exp \left(\alpha_{1} x-\beta / x\right)\left(\alpha_{1}+\beta / x^{2}\right)$ & 1 & {$\left[\alpha_{1}, \beta\right]$} \\
\hline Gompertz $(x \geq 0)|6|$ & $\beta^{-1}[\exp (\beta x)-1]$ & $\exp (\beta x)$ & $\alpha$ & $\beta$ \\
\hline Exponential power $(x \geq 0)|19|$ & $\exp \left[(\lambda x)^{\beta}\right]-1$ & $\beta \lambda \exp \left[(\lambda x)^{\beta}\right](\lambda x)^{\beta-1}$ & 1 & {$[\lambda, \beta]$} \\
\hline Chen $(x \geq 0)[4]$ & $\exp \left(x^{b}\right)-1$ & $b x^{b-1} \exp \left(x^{b}\right)$ & $\alpha$ & $b$ \\
\hline Pham $(x \geq 0)[16]$ & $\left(a^{x}\right)^{\beta}-1$ & $\beta\left(a^{x}\right)^{\beta} \log (a)$ & 1 & {$[a, \beta]$} \\
\hline
\end{tabular}

The survival function of the $\mathscr{G} \mathscr{E} \mathscr{W}$ family of distributions is given by

$$
S(x ; \delta, \alpha, \boldsymbol{\xi})=1-\gamma[\delta, \alpha H(x ; \boldsymbol{\xi})],
$$

$x>0$, and its hazard rate function becomes

$$
\tau(x ; \delta, \alpha, \boldsymbol{\xi})=\frac{\alpha^{\delta} h(x ; \boldsymbol{\xi}) H(x ; \boldsymbol{\xi})^{\delta-1} \exp [-\alpha H(x ; \boldsymbol{\xi})]}{\Gamma(\boldsymbol{\delta}) S(x ; \boldsymbol{\delta}, \alpha, \boldsymbol{\xi})},
$$

$x>0$.

\subsection{Expansions for the distribution and density functions}

Here, we derive useful expansions to obtain some important statistical quantities such as the noncentral moment, generating function and Rényi entropy. Raising the density function (2.2) to a positive power $s$ gives

$$
f(x ; \delta, \alpha, \boldsymbol{\xi})^{s}=\frac{\alpha^{s \delta}}{\Gamma(\boldsymbol{\delta})^{s}} h(x ; \boldsymbol{\xi})^{s} H(x ; \boldsymbol{\xi})^{s(\boldsymbol{\delta}-1)} \exp [-s \alpha H(x ; \boldsymbol{\xi})]
$$

For any real number $\delta>0$, we have the following equality (see http://functions . wolf ram.com/ElementaryFunctions/Log/06/01/04/03/)

$$
\{-\log [1-G(x ; \alpha, \boldsymbol{\xi})]\}^{\delta-1}=(\boldsymbol{\delta}-1) \sum_{k=0}^{\infty}\left(\begin{array}{c}
k+1-\delta \\
k
\end{array}\right) \sum_{j=0}^{k} \frac{(-1)^{j+k}\left(\begin{array}{l}
k \\
j
\end{array}\right) p_{j, k}}{(\boldsymbol{\delta}-1-j)} G(x ; \alpha, \boldsymbol{\xi})^{\alpha+k-1}
$$


Nascimento et al.

where the quantities $p_{j, k}$ can be obtained recursively, for $k=1,2, \ldots$, as

$$
p_{j, k}=\frac{1}{k} \sum_{m=1}^{k}[k-m(j+1)] c_{m} p_{j, k-m}
$$

$p_{j, 0}=1$ and $c_{k}=(-1)^{k+1} /(k+1)$. Applying (2.3) in equation (1.2) and using the binomial expansion, we can express 2.2] as an infinite linear combination of $\mathscr{E} \mathscr{W}$ densities. We have

$$
f(x ; \delta, \alpha, \boldsymbol{\xi})=\sum_{r=0}^{\infty} v_{r} g(x ; \alpha(r+1), \boldsymbol{\xi})
$$

where

$$
v_{r}=\frac{(-1)^{r}(\delta-1)}{(r+1) \Gamma(\delta)} \sum_{k=0}^{\infty} \sum_{j=0}^{k} \frac{(-1)^{j+k}}{(\boldsymbol{\delta}-1-j)}\left(\begin{array}{l}
k \\
j
\end{array}\right)\left(\begin{array}{c}
k+1-\delta \\
k
\end{array}\right)
$$

Equation (2.4) is the main result of this section.

\section{Moments, generating function and log-moment}

\subsection{Moments}

Let $X$ be a random variable following the $\mathscr{G} \mathscr{E} \mathscr{W}$ distribution with parameters $\delta, \alpha$ and $\boldsymbol{\xi}$, say $X \sim \mathscr{G} \mathscr{E} \mathscr{W}(\delta, \alpha, \xi)$. The $n$th noncentral moment is given by

$$
\mathrm{E}\left(X^{n}\right)=\int_{\mathscr{D}} x^{n} \frac{\alpha^{\delta-1}}{\Gamma(\boldsymbol{\delta})} H(x ; \boldsymbol{\xi})^{\delta-1} g(x ; \alpha, \boldsymbol{\xi}) \mathrm{d} x=\frac{\alpha^{\delta-1}}{\Gamma(\boldsymbol{\delta})} \mathrm{E}_{Y}\left[Y^{n} H(Y ; \boldsymbol{\xi})^{\delta-1}\right] .
$$

Here and henceforth $Y$, denotes a random variable following the $\mathscr{E} \mathscr{W}$ distribution with pdf given by (1.4). We can also rewrite (3.1) as

$$
\mathrm{E}\left(X^{n}\right)=\int_{\mathscr{D}} x^{n} f(x ; \boldsymbol{\delta}, \alpha, \boldsymbol{\xi}) \mathrm{d} x=\frac{\alpha^{\delta}}{\Gamma(\boldsymbol{\delta})} \int_{\mathscr{D}} x^{n} H(x ; \boldsymbol{\xi})^{\delta-1} h(x ; \boldsymbol{\xi}) \exp [-\alpha H(x ; \boldsymbol{\xi})] \mathrm{d} x
$$

Setting $u=H(x ; \boldsymbol{\xi})$, we have $\mathrm{d} u=h(x ; \boldsymbol{\xi}) \mathrm{d} x, x=H^{-1}(u ; \boldsymbol{\xi})$ and then

$$
\mathrm{E}\left(X^{n}\right)=\frac{\alpha^{\delta}}{\Gamma(\delta)} \int_{\mathscr{A}}\left[H^{-1}(u ; \xi)\right]^{n} u^{\delta-1} \exp (-\alpha u) \mathrm{d} u
$$

where $\mathscr{A}=\left\{u: H^{-1}(u ; \boldsymbol{\xi}) \in \mathscr{D}\right\}$. The integral in 3.2) can be obtained in closed-form for some special models.

In Table 2, we list the $H^{-1}(x ; \boldsymbol{\xi})$ function for some special cases. Table 3 provides $\mathrm{E}\left(X^{n}\right)$ for the exponential, Rayleigh, Weibull and Pareto distributions. 
Table 2. The $H^{-1}(x ; \boldsymbol{\xi})$ function

\begin{tabular}{lc}
\hline Distribution & $H^{-1}(x ; \boldsymbol{\xi})$ \\
\hline Exponential power & $\frac{[\log (x+1)]^{1 / \beta}}{\lambda}$ \\
Chen & {$[\log (x+1)]^{1 / \beta}$} \\
Weibull extension & $\lambda\left[\log \left(\frac{x}{\lambda}+1\right)\right]^{1 / \beta}$ \\
Log-Weibull & $\sigma \log (x)+\mu$ \\
Kies & $\frac{x^{1 / \beta} \sigma+\mu}{x^{1 / \beta}+1}$ \\
Gen. Power Weibull & $\beta\left[(x+1)^{1 / \theta}-1\right]^{1 / \alpha_{1}}$ \\
Gompertz & $\frac{\log (\beta x+1)}{\beta}$ \\
Pham & {$\left[\frac{\log (x+1)}{\log (a)}\right]^{1 / \beta}$} \\
\hline
\end{tabular}

Table 3. Values of $\mathrm{E}\left(X^{n}\right)$

\begin{tabular}{cccc}
\hline \multicolumn{3}{c}{$\mathrm{E}\left(X^{n}\right)$} \\
\hline Exponential & Rayleigh & Weibull & Pareto (for $k>\alpha$ ) \\
\hline$\frac{\Gamma(n+\delta)}{\alpha^{n} \Gamma(\delta)}$ & $\frac{\Gamma\left(\frac{n}{2}+\delta\right)}{\alpha^{\frac{n}{2}} \Gamma(\delta)}$ & $\frac{\Gamma\left(\frac{n}{\gamma}+\delta\right)}{\alpha^{\frac{n}{\gamma}} \Gamma(\delta)}$ & $\frac{\alpha^{\delta} k^{n}}{(k-\alpha)^{\delta}}$ \\
\hline
\end{tabular}

\subsection{Moment generating function}

In a similar manner, the moment generating function (mgf) of the $\mathscr{G} \mathscr{E} \mathscr{W}$ family of distributions is given by

$$
M(t)=\mathrm{E}\left(\mathrm{e}^{t X}\right)=\frac{\alpha^{\delta-1}}{\Gamma(\boldsymbol{\delta})} \mathrm{E}_{Y}\left[\mathrm{e}^{t X} H(Y ; \boldsymbol{\xi})^{\delta-1}\right]
$$

This equation can be expressed as

$$
M(t)=\frac{\alpha^{\delta}}{\Gamma(\boldsymbol{\delta})} \int_{\mathscr{D}} h(x ; \boldsymbol{\xi}) H(x ; \boldsymbol{\xi})^{\delta-1} \exp [-\alpha H(x ; \boldsymbol{\xi})+t x] \mathrm{d} x .
$$

Setting again $u=H(x ; \boldsymbol{\xi})$, we obtain $M(t)=\alpha^{\delta} \Gamma^{-1}(\delta) \int_{\mathscr{A}} u^{\delta-1} \exp \left[-\alpha u+t H^{-1}(u ; \boldsymbol{\xi})\right] \mathrm{d} u$.

\subsection{Log moment}

The $k$ th log-moment of the $\mathscr{G} \mathscr{E} \mathscr{W}$ family of distributions reduces to

$$
\begin{aligned}
\mathrm{E}\left[\log ^{k}(X)\right] & =\int_{\mathscr{D}} \log ^{k}(x) f(x ; \boldsymbol{\delta}, \alpha, \boldsymbol{\xi}) \mathrm{d} x=\frac{\alpha^{\delta-1}}{\Gamma(\boldsymbol{\delta})} \int_{\mathscr{D}} \log ^{k}(x) H(x ; \boldsymbol{\xi})^{\delta-1} g(x ; \alpha, \boldsymbol{\xi}) \mathrm{d} x \\
& =\frac{\alpha^{\delta-1}}{\Gamma(\boldsymbol{\delta})} \mathrm{E}_{Y}\left[\log ^{r}(Y) H(Y ; \boldsymbol{\xi})^{\delta-1}\right] .
\end{aligned}
$$

\subsection{Dependent-H moment}

Theorem 1. Let $Y$ and $X$ be two random variables represented by the cdf's (1.3) and 2.1), respectively. Thus, the following results hold: 
(1) $\mathrm{E}_{X}\left[H(X ; \boldsymbol{\xi})^{k}\right]=\frac{\Gamma(\delta+k)}{\alpha^{k} \Gamma(\delta)}$;

(2) $\mathrm{E}_{X}\left\{\log [H(X ; \boldsymbol{\xi})]^{k} H(X ; \boldsymbol{\xi})^{r-\delta+1}\right\}=\frac{\alpha^{\delta-1}}{\Gamma(\boldsymbol{\delta})} \frac{\partial^{k}}{\partial r^{k}}\left[\frac{\Gamma(r+1)}{\alpha^{r}}\right]$;

(3) $\mathrm{E}_{X}\left\{\log ^{k}[H(X ; \boldsymbol{\xi})]\right\}=\frac{\alpha^{\delta-1}}{\Gamma(\delta)} \frac{\partial^{k}}{\partial \delta^{k}}\left[\frac{\Gamma(\delta)}{\alpha^{\delta-1}}\right]$.

Setting $\delta=1$ in these equations, we obtain the corresponding expressions for the random variable $Y$. The proof of this theorem is given in the appendix B.

\subsection{Incomplete moments}

The $k$ th incomplete moment of a random variable $X$ following the $\mathscr{G} \mathscr{E} \mathscr{W}$ distribution is determined as

$$
T_{k}(z)=E\left(X^{k} \mid X<z\right)=\int_{-\infty}^{z} x^{k} f(x ; \delta, \alpha, \boldsymbol{\xi}) \mathrm{d} x=\sum_{r=0}^{\infty} v_{r} T_{k}^{\prime}(z)
$$

and then

$$
T_{k}(z)=\sum_{r=0}^{\infty} v_{r} T_{k}^{\prime}(z)
$$

where $T_{k}^{\prime}(z)=\int_{-\infty}^{z} x^{k} g(x ; \alpha(r+1), \xi) \mathrm{d} x$ is the $k$ th incomplete moment of the $\mathscr{E} \mathscr{W}$ distribution and the quantity $v_{r}$ is given in 2.5.

\section{Quantile function and random number generator}

The $\mathscr{G} \mathscr{E} \mathscr{W}$ quantile function can be expressed in terms of the quantile function of the gamma distribution and of the inverse function of $H$, which are denoted by $Q_{\Gamma}(\delta ; u)$ and $H^{-1}(\cdot)$, respectively. From the $\mathscr{G} \mathscr{E} \mathscr{W}$ cumulative distribution $F(x ; \delta, \alpha, \boldsymbol{\xi})=\gamma[\delta, \alpha H(x ; \boldsymbol{\xi})]$, we have $\gamma[\delta, \alpha H(x ; \boldsymbol{\xi})]=u$ and, as a consequence, $\alpha H(x ; \xi)=Q_{\Gamma}(\delta ; u)$. Therefore, the $\mathscr{G} \mathscr{E} \mathscr{W}$ quantile function can be expressed as

$$
Q(\delta, \alpha, \boldsymbol{\xi} ; u)=H^{-1}\left(\frac{Q_{\Gamma}(\delta ; u)}{\alpha} ; \boldsymbol{\xi}\right)
$$

For example, from Table 2, the quantile functions for the Chen and Pham distributions are given by

$$
\left[\log \left(Q_{\Gamma}(\delta, u)+1\right)\right]^{1 / \beta} \text { and }\left[\frac{\log \left(Q_{\Gamma}(\delta, u)+1\right)}{\log (a)}\right]^{1 / \beta} \text {, respectively. }
$$

Hence, the generator for $X \sim \mathscr{G} \mathscr{E} \mathscr{W}(\delta, \alpha, \xi)$ can be given by the following algorithm:

1: Generate $U \sim U(0,1)$.

2: Specify a function $H(\cdot ; \cdot)$ such as anyone in Table 1 .

3: Obtain a outcome of $X$ by $X=H^{-1}\left(\frac{Q_{\Gamma}(\delta ; U)}{\alpha}, \xi\right)$. 


\subsection{Expansion for the quantile function}

The quantile function of the $\mathscr{G} \mathscr{E} \mathscr{W}$ distribution can be expressed in terms of a power series of a transformed variable $v$, which takes the form $v=p(u-t)^{\rho}$, for $p, t$ and $\rho$ known constants,

$$
Q(u)=\sum_{i=0}^{\infty} m_{i} v^{i},
$$

where the coefficients $m_{i}$ are suitably chosen real numbers. In Steinbrecher and Shaw [20], for the gamma distribution with shape parameter $\delta>0$, equation $[4.2)$ is defined by $v=[\Gamma(\delta+1) u]^{1 / \delta}$ and

$$
m_{i}= \begin{cases}0, & \text { if } i=0 \\ 1, & \text { if } i=1 \\ a_{i+1}, & \text { if } i \geq 1\end{cases}
$$

where

$$
a_{i+1}=\frac{1}{i(\boldsymbol{\delta}+i)}\left\{\sum_{r=1}^{i} \sum_{s=1}^{i-s+1} a_{r} a_{s} a_{i-r-s+2} s(i-r-s+2)-\Delta(i) \sum_{r=2}^{i} a_{r} a_{i-r+2} r[r-\delta-(1-\delta)(i+2-r)]\right\},
$$

$\Delta(i)=0$ if $i<2$ and $\Delta(i)=1$ if $i \geq 2$. In this case, the first coefficients are $a_{2}=1 /(\delta+1)$, $a_{3}=(3 \delta+5) /\left[2(\delta+1)^{2}(\delta+2)\right], \ldots$ Hence, the power series for the gamma quantile function can be expressed as

$$
Q_{\Gamma}(\delta ; u)=\sum_{i=0}^{\infty} m_{i} \Gamma(\delta+1)^{i / \delta} u^{i / \delta} .
$$

Applying (4.3) to equation (4.1), it follows the $\mathscr{G} \mathscr{E} \mathscr{W}$ quantile function

$$
Q(\delta, \alpha, \xi ; u)=H^{-1}\left(\frac{1}{\alpha} \sum_{i=0}^{\infty} m_{i} \Gamma(\delta+1)^{i / \delta} u^{i / \delta} ; \boldsymbol{\xi}\right)
$$

\subsection{Skewness and kurtosis}

There are several robust measures in the literature for location and dispersion. The median, for example, can be used for location and the interquartile range. Both the median and the interquartile range are based on quantiles. From this fact, Bowley [2] proposed a coefficient of skewness based on quantiles given by

$$
S K=\frac{Q(3 / 4)+Q(1 / 4)-2 Q(1 / 2)}{Q(3 / 4)-Q(1 / 4)},
$$

where $Q(\cdot)$ is the quantile function of a given distribution. It can be shown that Bowley's coefficient of skewness takes the value zero for symmetric distributions. Additionally, its largest value is one and the lowest is -1 .

Moors [12] demonstrated that the conventional measure of kurtosis may be interpreted as a dispersion around the values $\mu+\sigma$ and $\mu-\sigma$. Thus, the probability mass focuses around $\mu$ or on the tails of the distribution. Therefore, based on this interpretation, Moors [12] proposed, as an alternative to the conventional coefficient of kurtosis, a robust measure based on octiles given by

$$
K R=\frac{[Q(7 / 8)-Q(5 / 8)]+[Q(3 / 8)-Q(1 / 8)]}{Q(6 / 8)-Q(2 / 8)} .
$$




\section{Order statistics}

In the following discussion, we derive the order statistics and their moments. The pdf of the $i$ th order statistic $X_{i: n}$, for $i=1,2, \ldots, n$, can be expressed as

$$
f_{i: n}(x)=\frac{f(x)}{B(i, n-i+1)} F^{i-1}(x)[1-F(x)]^{n-i}=\frac{f(x)}{B(i, n-i+1)} \sum_{k=0}^{n-i}(-1)^{k}\left(\begin{array}{c}
n-i \\
k
\end{array}\right) F^{i+k-1}(x) .
$$

From equation (C.2) given in Appendix C, we obtain

$$
f_{i: n}(x)=\frac{g(x)}{B(i, n-i+1)} \sum_{k=0}^{n-i}(-1)^{k}\left(\begin{array}{c}
n-i \\
k
\end{array}\right) \sum_{m=0}^{\infty} \eta_{m, v} H(x ; \boldsymbol{\xi})^{m+\delta(i+k)-1},
$$

where $\eta_{m, v}=\alpha^{\delta-1} s_{m, v} / \Gamma(\delta)$ and $s_{m, v}$ is defined in this appendix. Additionally, from equation (C.2) given in Appendix C, the $v$ th ordinary moment of $X_{i: n}$ becomes

$$
\mathrm{E}\left(X_{i: n}^{v}\right)=\int_{\mathscr{D}} x^{v} f_{i: n}(x) \mathrm{d} x=\frac{1}{B(i, n-i+1)} \sum_{k=0}^{n-i}(-1)^{k}\left(\begin{array}{c}
n-i \\
k
\end{array}\right) \mu_{v, i+k-1}
$$

where the quantity $\mu_{v, i+k-1}=\mathrm{E}\left[X^{v} F^{i+k-1}(X)\right]$ is the probability weighted moment (pwm) of the $\mathscr{G} \mathscr{E} \mathscr{W}$ distribution.

\section{Mean deviations}

The mean deviations about the mean and the median for the $\mathscr{G} \mathscr{E} \mathscr{W}$ family of distributions can be expressed as

$$
\delta_{1}(X)=\int_{\mathscr{D}}|x-\mu| f(x ; \delta, \alpha, \boldsymbol{\xi}) \mathrm{d} x \quad \text { and } \quad \delta_{2}(X)=\int_{\mathscr{D}}|x-M| f(x ; \delta, \alpha, \boldsymbol{\xi}) \mathrm{d} x,
$$

respectively, where $\mu=\mathrm{E}(X)$ denotes the mean and $M=\operatorname{Median}(X)$ the median. The median follows from the nonlinear equation $F(M ; \delta, \alpha, \xi)=1 / 2$. These quantities can be reduced to

$$
\delta_{1}(X)=2 \mu F(\mu ; \delta, \alpha, \xi)-2 T_{1}(\mu) \quad \text { and } \quad \delta_{2}(X)=\mu-2 T_{1}(M),
$$

where $T_{1}(z)=\int_{-\infty}^{z} x f(x ; \delta, \alpha, \boldsymbol{\xi}) \mathrm{d} x$ is the first incomplete moment.

From equation (3.3), the quantity $T_{1}(z)$ for the $\mathscr{G} \mathscr{E} \mathscr{W}$ distribution becomes $T_{1}(z)=$ $\sum_{r=0}^{\infty} v_{r} T_{1}^{\prime}(z)$, where $T_{1}^{\prime}(z)=\int_{-\infty}^{z} x g(x ; \alpha(r+1), \xi) \mathrm{d} x$ is the first incomplete moment of the $\mathscr{E} \mathscr{W}$ distribution.

\section{Rényi entropy}

Let $Y$ be a random variable with density $f(y ; \theta)$ with support $y \in \mathscr{A} \subset \mathbb{R}$. The Rényi entropy is defined by

$$
H_{\mathrm{R}}^{s}(Y)=\frac{1}{1-s} \log \left\{\mathrm{E}_{Y}\left[f(Y ; \theta)^{s-1}\right]\right\}=\frac{1}{1-s} \log \left(\int_{\mathscr{A}} f(y ; \theta)^{s} \mathrm{~d} y\right),
$$

where $s \in(0, \infty) \backslash\{1\}$. 
We can obtain the Rényi entropy for the $\mathscr{G} \mathscr{E} \mathscr{W}$ distribution as

$$
\begin{aligned}
H_{\mathrm{R}}^{s}(X) & =\frac{1}{1-s} \log \left(\frac{\alpha^{s \delta}}{\Gamma(\boldsymbol{\delta})^{s}} \int_{\mathscr{D}} H(x ; \boldsymbol{\xi})^{s(\delta-1)} h(x ; \boldsymbol{\xi})^{s} \exp [-s \alpha H(x ; \boldsymbol{\xi})] \mathrm{d} x\right) \\
& =\frac{1}{1-s}\left\{s \delta \log (\alpha)-s \log [\Gamma(\boldsymbol{\delta})]+\log \left(\int_{\mathscr{D}} H(x ; \boldsymbol{\xi})^{s(\delta-1)} h(x ; \boldsymbol{\xi})^{s} \exp [-s \alpha H(x ; \boldsymbol{\xi})] \mathrm{d} x\right)\right\} .
\end{aligned}
$$

\section{Shannon entropy}

Let $Y$ be defined as in Section 7 . Here, we derive the Shannon entropy defined by

$$
H_{\mathrm{S}}(Y)=\mathrm{E}_{Y}\{-\log [f(Y ; \theta)]\}=-\int_{\mathscr{D}} \log [f(y ; \theta)] f(y ; \theta) \mathrm{d} y .
$$

The log-likelihood function relative to one observation follows from $(2.2)$ as

$$
\log [f(x ; \delta, \alpha, \boldsymbol{\xi})]=\log \left[\frac{\alpha^{\delta}}{\Gamma(\boldsymbol{\delta})}\right]+(\delta-1) \log [H(x ; \boldsymbol{\xi})]-\alpha H(x ; \boldsymbol{\xi})+\log [h(x ; \boldsymbol{\xi})] .
$$

Thus, the Shannon entropy of $X$ can be expressed as

$$
H_{\mathrm{S}}(X)=-\log \left[\frac{\alpha^{\delta}}{\Gamma(\boldsymbol{\delta})}\right]-(\delta-1) \mathrm{E}_{X}\{\log [H(X ; \boldsymbol{\xi})]\}+\alpha \mathrm{E}_{X}[H(X ; \boldsymbol{\xi})]-\mathrm{E}_{X}\{\log [h(X ; \boldsymbol{\xi})]\}
$$

Using Theorem 1 , the following results hold: (i) $\mathrm{E}_{X}\{\log [H(X ; \boldsymbol{\xi})]\}=\psi(\delta)-\log (\alpha)$, where $\psi(\cdot)$ is the digamma function and (ii) $\mathrm{E}_{X}[H(X ; \boldsymbol{\xi})]=\delta / \alpha$. Finally, the Shannon entropy reduces to

$$
H_{\mathrm{S}}(X)=\log \left[\frac{\Gamma(\delta)}{\alpha}\right]-(\delta-1) \psi(\delta)+\delta-\mathrm{E}_{X}\{\log [h(X ; \boldsymbol{\xi})]\} .
$$

\section{Cross entropy and Kullback-Leibler Divergence}

Let $X$ and $Y$ be two random variables with common support $\mathbb{R}_{+}$whose densities are $f_{X}\left(x ; \theta_{1}\right)$ and $f_{Y}\left(y ; \theta_{2}\right)$, respectively. Cover and Thomas [5] defined the cross entropy as

$$
C_{X}(Y)=\mathrm{E}_{X}\left\{-\log \left[f_{Y}\left(X ; \theta_{2}\right)\right]\right\}=-\int_{0}^{\infty} f_{X}\left(z ; \theta_{1}\right) \log \left[f_{Y}\left(z ; \theta_{2}\right)\right] \mathrm{d} z
$$

Now, consider $X \sim \mathscr{G} \mathscr{E} \mathscr{W}\left(\delta_{x}, \alpha_{x}, \boldsymbol{\xi}_{x}\right)$ and $Y \sim \mathscr{G} \mathscr{E} \mathscr{W}\left(\delta_{y}, \alpha_{y}, \boldsymbol{\xi}_{y}\right)$. After some algebraic manipulations, we obtain

$$
\begin{aligned}
C_{X}(Y) & =-\int_{\mathscr{D}} f_{X}\left(z ; \delta_{x}, \alpha_{x}, \boldsymbol{\xi}_{x}\right) \log \left[f_{Y}\left(z ; \delta_{y}, \alpha_{y}, \boldsymbol{\xi}_{y}\right)\right] \mathrm{d} z \\
& =-\left\{\log \left[\frac{\alpha_{y}^{\delta_{y}}}{\Gamma\left(\boldsymbol{\delta}_{y}\right)}\right]+\delta_{y} \mathrm{E}_{X}\left\{\log \left[H\left(X ; \boldsymbol{\xi}_{y}\right)\right]\right\}-\alpha_{y} \mathrm{E}_{X}\left[H\left(X ; \boldsymbol{\xi}_{y}\right)\right]+\mathrm{E}_{X}\left\{\log \left[h\left(X ; \boldsymbol{\xi}_{y}\right)\right]\right\}\right\} .
\end{aligned}
$$


Nascimento et al.

An important measure in statistical information theory is the Kullback-Leibler divergence given by

$$
D(X \| Y)=C_{X}(Y)-H_{\mathrm{S}}(X)=\mathrm{E}_{X}\left\{\log \left[\frac{f_{X}\left(X ; \delta_{x}, \alpha_{x}, \boldsymbol{\xi}_{x}\right)}{f_{Y}\left(X ; \delta_{y}, \alpha_{y}, \boldsymbol{\xi}_{y}\right)}\right]\right\}
$$

Applying (8.1) and 9.1) in equation 9.2) yields

$$
\begin{aligned}
D(X \| Y)= & \mathrm{E}_{X}\left\{\log \left[\frac{h\left(X ; \boldsymbol{\xi}_{x}\right)}{h\left(X ; \boldsymbol{\xi}_{y}\right)}\right]\right\}+\log \left[\frac{\Gamma\left(\delta_{y}\right) \alpha_{x}}{\alpha_{y}^{\delta_{y}} \Gamma\left(\delta_{x}\right)}\right]-\delta_{y} \mathrm{E}_{X}\left[\log H\left(X ; \boldsymbol{\xi}_{y}\right)\right]+\left(\delta_{x}-1\right) \psi\left(\delta_{x}\right) \\
& -\delta_{x}+\alpha_{y} \mathrm{E}_{X}\left[H\left(X ; \boldsymbol{\xi}_{y}\right)\right] .
\end{aligned}
$$

\section{Estimation and observed information matrix}

The parameters of the $\mathscr{G} \mathscr{E} \mathscr{W}$ distribution can be estimated by the method of maximum likelihood. Let $x_{1}, \cdots, x_{n}$ be a random sample of size $n$ from $X \sim \mathscr{G} \mathscr{E} \mathscr{W}(\delta, \alpha, \xi)$. The log-likelihood function for the vector of parameters $\boldsymbol{\theta}=\left(\delta, \alpha, \boldsymbol{\xi}^{\top}\right)^{\top}$ can be written as

$$
l(\boldsymbol{\theta})=n \delta \log (\alpha)-n \log [\Gamma(\boldsymbol{\delta})]+(\boldsymbol{\delta}-1) \sum_{i=1}^{n} \log \left[H\left(x_{i} ; \boldsymbol{\xi}\right)\right]-\alpha \sum_{i=1}^{n} H\left(x_{i} ; \boldsymbol{\xi}\right)+\sum_{i=1}^{n} \log \left[h\left(x_{i} ; \boldsymbol{\xi}\right)\right] .
$$

The components of the score vector $\mathbf{U}(\theta)$ are

$$
U_{\delta}(\boldsymbol{\theta})=\frac{\partial l(\boldsymbol{\theta})}{\partial \delta}=-n \psi(\boldsymbol{\delta})+\sum_{i=1}^{n} \log \left[H\left(x_{i} ; \boldsymbol{\xi}\right)\right]+n \log \alpha, \quad U_{\alpha}(\boldsymbol{\theta})=\frac{\partial l(\theta)}{\partial \alpha}=\frac{n \delta}{\alpha}-\sum_{i=1}^{n} H\left(x_{i} ; \boldsymbol{\xi}\right)
$$

and $U_{\boldsymbol{\xi}_{k}}(\boldsymbol{\theta})=\frac{\partial l(\boldsymbol{\theta})}{\partial \boldsymbol{\xi}_{k}}=(\boldsymbol{\delta}-1) \sum_{i=1}^{n} \frac{1}{H\left(x_{i} ; \boldsymbol{\xi}\right)} \frac{\partial H\left(x_{i} ; \boldsymbol{\xi}\right)}{\partial \boldsymbol{\xi}_{k}}-\alpha \sum_{i=1}^{n} \frac{\partial H\left(x_{i} ; \boldsymbol{\xi}\right)}{\partial \boldsymbol{\xi}_{k}}+\sum_{i=1}^{n} \frac{1}{h\left(x_{i} ; \boldsymbol{\xi}\right)} \frac{\partial h\left(x_{i} ; \boldsymbol{\xi}\right)}{\partial \boldsymbol{\xi}_{k}}$.

The partitioned observed information matrix for the $\mathscr{G} \mathscr{E} \mathscr{W}$ distribution is

$$
J(\boldsymbol{\theta})=-\left(\begin{array}{cccc}
U_{\delta \delta} & U_{\delta \alpha} & \mid & U_{\delta \boldsymbol{\xi}}^{\top} \\
U_{\alpha \delta} & U_{\alpha \alpha} & \mid & U_{\alpha \boldsymbol{\xi}}^{\top} \\
-- & -- & -- & -- \\
U_{\delta \boldsymbol{\xi}} & U_{\alpha \boldsymbol{\xi}} & \mid & U_{\boldsymbol{\xi} \xi}
\end{array}\right)
$$

whose elements are $U_{\delta \delta}(\boldsymbol{\theta})=-\psi^{(1)}(\boldsymbol{\delta}), U_{\delta \alpha}(\boldsymbol{\theta})=n \alpha^{-1}, U_{\alpha \alpha}(\boldsymbol{\theta})=-n \delta \alpha^{-2}$,

$$
\begin{aligned}
U_{\delta \boldsymbol{\xi}_{k}}(\boldsymbol{\theta}) & =\sum_{i=1}^{n} \frac{1}{H\left(x_{i} ; \boldsymbol{\xi}\right)} \frac{\partial H\left(x_{i} ; \boldsymbol{\xi}\right)}{\partial \boldsymbol{\xi}_{k}}, \quad U_{\alpha \boldsymbol{\xi}_{k}}(\boldsymbol{\theta})=\sum_{i=1}^{n} \frac{\partial H\left(x_{i} ; \boldsymbol{\xi}\right)}{\partial \boldsymbol{\xi}_{k}}, \quad \text { and } \\
U_{\boldsymbol{\xi}_{k} \boldsymbol{\xi}_{j}}(\boldsymbol{\theta})= & (\boldsymbol{\delta}-1) \sum_{i=1}^{n} \frac{1}{H\left(x_{i} ; \boldsymbol{\xi}\right)}\left[\frac{\partial^{2} H\left(x_{i} ; \boldsymbol{\xi}\right)}{\partial \boldsymbol{\xi}_{k} \boldsymbol{\xi}_{j}}-\frac{1}{H\left(x_{i} ; \boldsymbol{\xi}\right)} \frac{\partial H\left(x_{i} ; \boldsymbol{\xi}\right)}{\partial \boldsymbol{\xi}_{k}} \frac{\partial H\left(x_{i} ; \boldsymbol{\xi}\right)}{\partial \boldsymbol{\xi}_{j}}\right] \\
& -\alpha \sum_{i=1}^{n} \frac{\partial^{2} H\left(x_{i} ; \boldsymbol{\xi}\right)}{\partial \boldsymbol{\xi}_{k} \boldsymbol{\xi}_{j}}+\sum_{i=1}^{n} \frac{1}{h\left(x_{i} ; \boldsymbol{\xi}\right)}\left[\frac{\partial^{2} h\left(x_{i} ; \boldsymbol{\xi}\right)}{\partial \boldsymbol{\xi}_{k} \boldsymbol{\xi}_{j}}-\frac{1}{h\left(x_{i} ; \boldsymbol{\xi}\right)} \frac{\partial h\left(x_{i} ; \boldsymbol{\xi}\right)}{\partial \boldsymbol{\xi}_{k}} \frac{\partial h\left(x_{i} ; \boldsymbol{\xi}\right)}{\partial \boldsymbol{\xi}_{j}}\right] .
\end{aligned}
$$




\section{Two special models}

\subsection{The gamma modified Weibull distribution}

For $H(x ; \gamma)=x^{\gamma} \exp (\lambda x)$ and $h(x ; \gamma)=x^{\gamma-1} \exp (\lambda x)(\gamma+\lambda x)$, we obtain the gamma modified Weibull ( $\mathscr{G} \mathscr{M} \mathscr{W})$ density $f(x ; \delta, \alpha, \gamma, \lambda)=\alpha^{\delta} \Gamma^{-1}(\delta) x^{\gamma \delta-1}(\gamma+\lambda x) \exp \left[\delta \lambda x-\alpha x^{\gamma} \exp (\lambda x)\right]$, where $x>0$ and $\lambda, \gamma \geq 0$. If $\delta=1$, it gives as special case the modified Weibull ( $\mathscr{M} \mathscr{W}$ ) distribution proposed by Lai et al. [11]. In addition, when $\lambda=0$, it gives the Weibull distribution.

$\alpha=0.1, \delta=2$ and $\lambda=0.5$

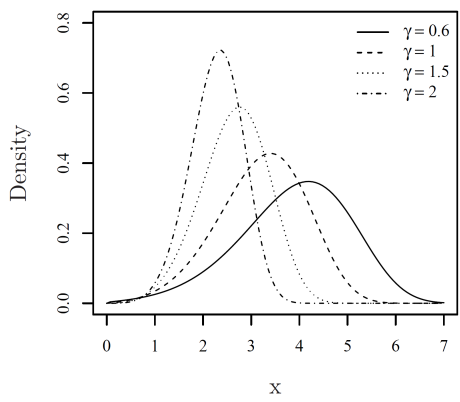

(a)

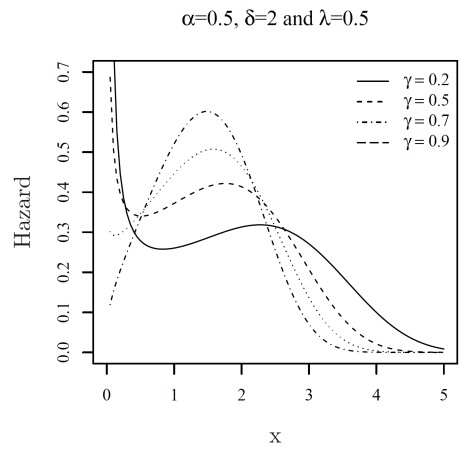

(c) $\alpha=9, \delta=1$ and $\lambda=0.5$

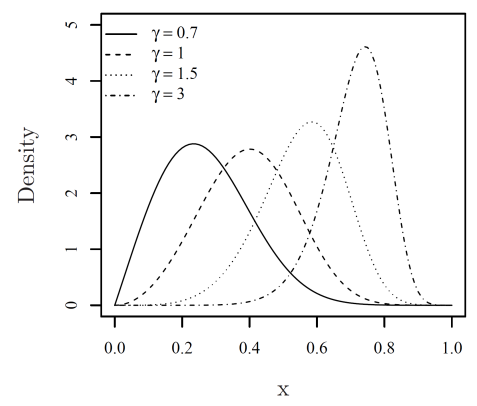

(b)

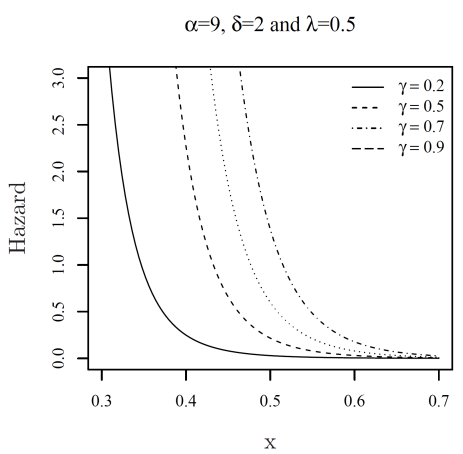

(d)

Fig. 1. Plots of the $\mathscr{G} \mathscr{M} \mathscr{W}$ density and Hazard rate function for some parameter values.

From equation (2.4), we can obtain $f(x ; \delta, \alpha, \gamma, \lambda)=\sum_{r=1}^{\infty} v_{r} g(x ; \alpha(r+1), \gamma, \lambda)$, where $g(x ; \alpha(r+1), \gamma, \lambda)$ is the $\mathscr{M} \mathscr{W}$ density function with parameters $\alpha(r+1), \gamma$ and $\lambda$. The $\mathscr{G} \mathscr{M} \mathscr{W}$ hazard function is $\tau(x ; \delta, \alpha, \gamma, \lambda)=[\Gamma(\delta) S(x ; \delta, \alpha, \gamma, \lambda)]^{-1} \alpha^{\delta}(\gamma+\lambda x) x^{\gamma \delta-1} \exp \left(\delta \lambda x-\alpha x^{\gamma} \mathrm{e}^{\lambda x}\right)$, where $x>0$.

The raw moment of a random variable $X$ following the $\mathscr{G} \mathscr{M} \mathscr{W}$ distribution has closed-form computed from (2.4) as

$$
\mathrm{E}\left(X^{k}\right)=\sum_{r=0}^{\infty} v_{r} \mu_{k}^{\prime}(r)
$$

where $\mu_{k}^{\prime}(r)=\int_{0}^{\infty} x^{k} g(x ; \alpha(r+1), \gamma, \lambda) \mathrm{d} x$ denotes the raw moment of the $\mathscr{M} \mathscr{W}$ distribution with parameters $\alpha(r+1), \gamma$ and $\lambda$. Carrasco et al. (2008) [3] obtained an infinite representation for this 
moment as

$$
\mu_{k}^{\prime}(r)=\sum_{i_{1}, \ldots, i_{k}=1}^{\infty} \frac{A_{i_{1}, \ldots, i_{k}} \Gamma\left(s_{k} / \gamma+1\right)}{[\alpha(r+1)]^{s_{k} / \gamma}}
$$

where $A_{i_{1}, \ldots, i_{k}}=a_{i_{1}}, \ldots, a_{i_{k}} \quad$ and $\quad s_{k}=i_{1}, \ldots, i_{k}$, and $a_{i}=\frac{(-1)^{i+1} i^{i-2}}{(i-1) !}\left(\frac{\lambda}{\gamma}\right)^{i-1}$.

Hence, the moments of the $\mathscr{G} \mathscr{M} \mathscr{W}$ distribution can be computed directly from equations 11.1 and (11.2).

\subsection{The gamma Pareto distribution}

For $H(x ; k)=\log (x / k)$ and $h(x ; k)=1 / x$, we obtain the gamma Pareto $(\mathscr{G} \mathscr{P})$ density

$$
f(x ; \delta, \alpha, k)=\frac{\alpha^{\delta} k^{\alpha}}{\Gamma(\delta) x^{\alpha+1}}\left[\log \left(\frac{x}{k}\right)\right]^{\delta-1}, x \geq k .
$$

The hazard rate function is

$$
\tau(x ; \delta, \alpha, k)=\frac{\alpha^{\delta} k^{\alpha}[\log (x / k)]^{\delta-1}}{\Gamma(\delta) x^{\alpha+1} S(x ; \delta, \alpha, k)} .
$$

From equations (2.4) and (11.3), we obtain the sth ordinary moment of $X$

$$
\mathrm{E}\left(X^{s}\right)=\alpha k^{s} \sum_{r=0}^{\infty} \frac{v_{r}(r+1)}{[\alpha(r+1)-s]}, \quad \text { for } \quad \alpha>s,
$$

where the coefficients $v_{r}$ are given by (2.5).

From equation $(7.1)$ we obtain the Rényi entropy of the $\mathscr{G} \mathscr{P}$ distribution, which is valid for $s>1$, as

$$
H_{\mathrm{R}}^{s}(X)=\frac{1}{1-s}\left\{s \delta \log (\alpha)-s \log \left[k^{s \alpha} \int_{k}^{\infty} x^{1-s \delta} \log ^{s(\delta-1)}(x / k) \mathrm{d} x\right]\right\}
$$

Similarly, we obtain from equation 8.1 the Shannon entropy given by $H_{\mathrm{S}}(X)=\log \left[\frac{\Gamma(\delta)}{\alpha}\right]-$ $(\delta-1) \psi(\delta)+\delta+\alpha \delta k^{\alpha-\delta}$.

\section{Application}

We assess the efficiency of the proposed model in an analysis of real data. We compare the fits of some $\mathscr{G} \mathscr{E} \mathscr{W}$ distributions and those of some sub-models such as the $\mathscr{G} \mathscr{M} \mathscr{W}$, gamma Weibull $(\mathscr{G} \mathscr{W})$, gamma Rayleigh $(\mathscr{G} \mathscr{R}), \mathscr{G} \mathscr{P}, \mathscr{M} \mathscr{W}$ and Weibull distributions. In order to estimate the parameters of these submodels in the class of the $\mathscr{G} \mathscr{E} \mathscr{W}$ distributions, we adopt the maximum likelihood method (as discussed in Section 10) using the subroutine NLMixed of the software SAS. The data for this application, consisting of the failure times of 20 mechanical components given in Murthy et al. [13] are listed in Table 4 .

Table 6 displays the MLEs of the parameters and the values of the statistics: Akaike Information Criterion (AIC), Bayesian Information Criterion (BIC) and Consistent Akaike Information Criterion (CAIC). From the values of these statistics, we verify that the $\mathscr{G} \mathscr{P}$ model provides a better fit to 
$\delta=0.9, \mathrm{k}=1$

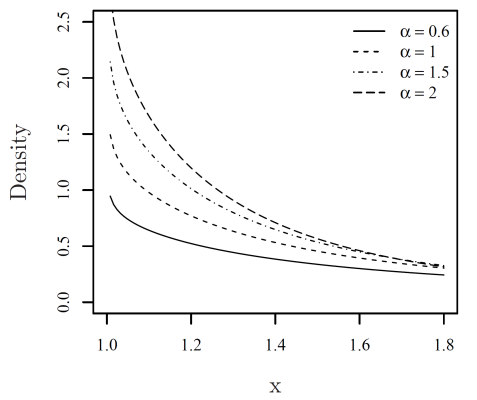

(a)

$\delta=0.8, \mathrm{k}=1$

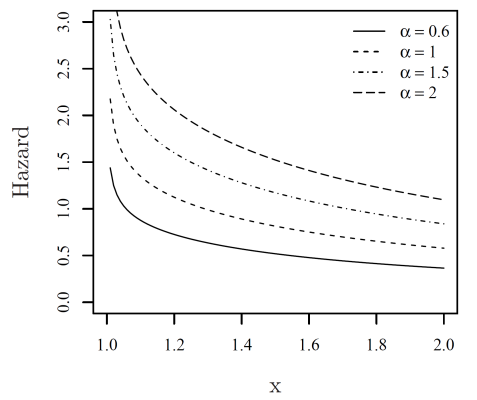

(c) $\delta=2, \mathrm{k}=1$

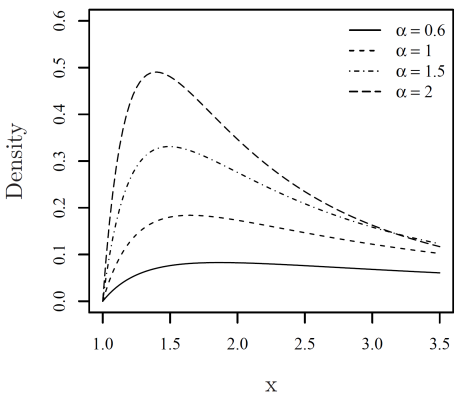

$\delta=1.2, \mathrm{k}=1$

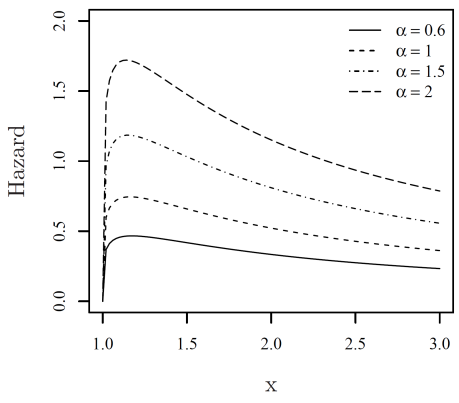

Fig. 2. Plots of the $\mathscr{G} \mathscr{P}$ density and hazard rate function for some parameter values.

Table 4. The failure times of 20 mechanical components

\begin{tabular}{llllllllll}
\hline 0.067 & 0.068 & 0.076 & 0.081 & 0.084 & 0.085 & 0.085 & 0.086 & 0.089 & 0.098 \\
0.098 & 0.114 & 0.114 & 0.115 & 0.121 & 0.125 & 0.131 & 0.149 & 0.160 & 0.485 \\
\hline
\end{tabular}

Table 5. The K-S statistics and $-2 \ell(\widehat{\theta})$ for some fitted models

\begin{tabular}{lcc}
\hline Model & $\mathrm{K}-\mathrm{S}$ & $-2 \ell(\widehat{\theta})$ \\
\hline $\mathscr{G} \mathscr{M} \mathscr{W}$ & 0.8761 & -71.2 \\
$\mathscr{G} \mathscr{W}$ & 0.1855 & -65.4 \\
$\mathscr{G} \mathscr{R}$ & 0.3001 & -50.7 \\
$\mathscr{G} \mathscr{P}$ & 0.2518 & -80.4 \\
$\mathscr{M} \mathscr{W}$ & 0.8007 & -61.7 \\
Weibull & 0.2641 & -52.8 \\
\hline
\end{tabular}

these data. Additionally, the $\mathscr{G} \mathscr{E} \mathscr{W}$ and $\mathscr{G} \mathscr{W}$ models are much better than the $\mathscr{M} \mathscr{W}$ and Weibull models. 


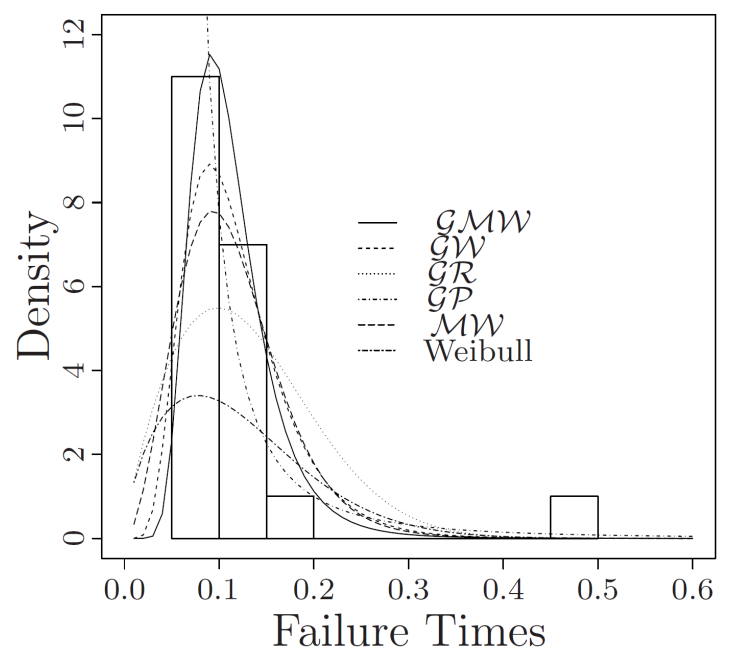

(a)

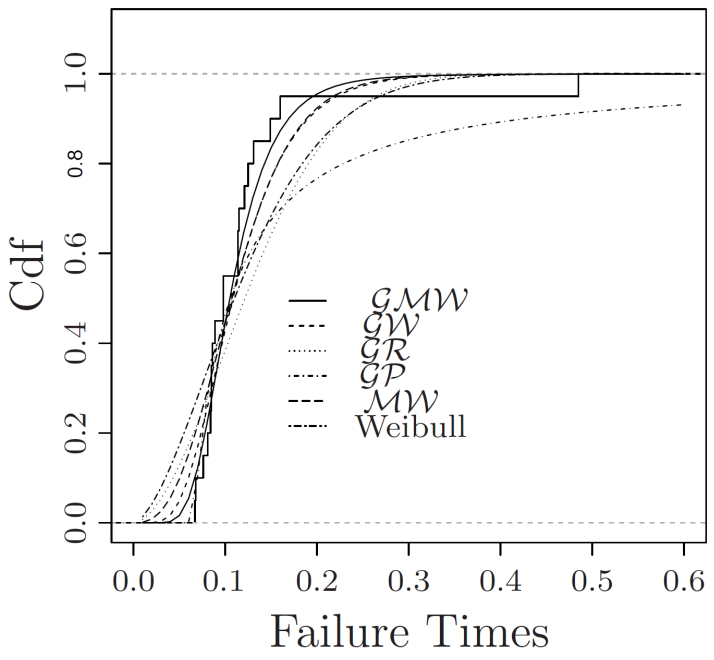

(b)

Fig. 3. Estimated densities, cumulative and empirical distributions from the fitted $\mathscr{G} \mathscr{M} \mathscr{W}, \mathscr{G} \mathscr{M}, \mathscr{G} \mathscr{R}, \mathscr{G} \mathscr{P}, \mathscr{M} \mathscr{W}$ and Weibull models for the failure times.

Table 6. MLEs of the model parameters and the statistics AIC, BIC and AICC

\begin{tabular}{|c|c|c|c|c|c|c|c|}
\hline Model & & Estimates & & & AIC & BIC & AICC \\
\hline $\mathscr{G} \mathscr{M}^{\mathscr{W}}(\delta, \alpha, \gamma, \lambda)$ & 93.9631 & 223.2331 & 0.3563 & -0.6258 & -63.2 & -59.2 & -60.5 \\
\hline $\mathscr{G} \mathscr{W}(\delta, \alpha, \gamma)$ & 72.2393 & 128.7642 & 0.2622 & & -59.4 & -57.9 & -56.4 \\
\hline $\mathscr{G} \mathscr{R}(\delta, \alpha)$ & 0.8884 & 39.7374 & & & -46.7 & -44.8 & -46.0 \\
\hline $\mathscr{G} \mathscr{P}(\delta, \alpha, k)$ & 0.7490 & 1.5826 & 0.0670 & & -76.4 & -74.4 & -75.6 \\
\hline$\overline{\mathscr{M} \mathscr{W}(\alpha, \gamma, \lambda)}$ & 828.48 & 2.9129 & -5.4296 & & -55.7 & -52.7 & $\overline{-54.2}$ \\
\hline Weibull $(\alpha, \gamma)$ & 25.9723 & 1.6422 & & & -48.8 & -46.9 & -48.1 \\
\hline
\end{tabular}

More information is provided by a visual comparison of the fitted density functions and the histogram of the data. The plots of the fitted $\mathscr{G} \mathscr{M} \mathscr{W}, \mathscr{G} \mathscr{W}, \mathscr{G} \mathscr{R}, \mathscr{G} \mathscr{P}, \mathscr{M} \mathscr{W}$ and Weibull density functions and estimated cumulative functions are given in Figure 3 . Based on these plots, we conclude that the new distributions provide adequate fits. Table 5 lists the values of the Kolmogorov-Smirnov $(\mathrm{K}-\mathrm{S})$ statistic and of $-2 \ell(\widehat{\theta})$.

\section{Conclusion}

We propose and study the gamma extended Weibull $(\mathscr{G} \mathscr{E} \mathscr{W})$ family of distributions. The new density function can be expressed as a mixture of extended Weibull density functions. This result is important to derive some mathematical properties of the new family including moments, generating function, mean deviations, Shannon entropy, Rényi entropy, Cross entropy and Kullback-Leibler Divergence. We also derive the density function of the order statistics and their moments. Two special distributions are investigated in some detail. The model parameters are estimated by maximum likelihood. An example to real data illustrates the importance and potentiality of the new family. 


\section{Appendix A. Theoretical background}

For a positive integer $s$, we have that (see Gradshteyn and Ryzhik [7])

$$
\left(\sum_{m=0}^{\infty} a_{m} x^{m}\right)^{s}=\sum_{m=0}^{\infty} t_{s, m} x^{m},
$$

where the coefficients $t_{s, m}$, for $m=1,2, \ldots$, are obtained by the recurrence equation $t_{s, 0}=a_{0}^{s}$ and $t_{s, m}=\left(m a_{0}\right)^{-1} \sum_{j=1}^{m}[j(s+1)-m] a_{j} t_{s, m-j}$.

\section{Appendix B. Proof of Theorem 1}

From equation (2.2), we have

$$
\int_{\mathscr{D}} H(x ; \boldsymbol{\xi})^{r} g(x ; \alpha, \boldsymbol{\xi}) \mathrm{d} x=\frac{\Gamma(r+1)}{\alpha^{r}} .
$$

The $k$ th derivative with respect to $r$ at both sides of equation (B.1) yields

$$
\int_{\mathscr{D}} \log ^{k}[H(x ; \boldsymbol{\xi})] H(x ; \boldsymbol{\xi})^{r} g(x ; \alpha, \boldsymbol{\xi}) \mathrm{d} x=\frac{\partial^{k}}{\partial r^{k}}\left[\frac{\Gamma(r+1)}{\alpha^{r}}\right]
$$

and then $\mathrm{E}\left\{\log ^{k}[H(X ; \boldsymbol{\xi})] H(X ; \boldsymbol{\xi})^{r-\delta+1}\right\}=\frac{\alpha^{\delta-1}}{\Gamma(\delta)} \frac{\partial^{k}}{\partial r^{k}}\left[\frac{\Gamma(r+1)}{\alpha^{r}}\right]$.

Using equation (B.1) with $r=\delta-1$ and differentiating $k$ times with respect to $\delta$,

$$
\int_{\mathscr{D}} \log ^{k}[H(x ; \boldsymbol{\xi})] H(x ; \boldsymbol{\xi})^{\delta-1} g(x ; \alpha, \boldsymbol{\xi}) \mathrm{d} x=\frac{\partial^{k}}{\partial \delta^{k}}\left[\frac{\Gamma(\boldsymbol{\delta})}{\alpha^{\delta-1}}\right] .
$$

After multiplying both sides of this equation by $\frac{\alpha^{\delta-1}}{\Gamma(\delta)}$, we can write $\mathrm{E}\left\{\log ^{k}[H(X ; \boldsymbol{\xi})]\right\}=$ $\frac{\alpha^{\delta-1}}{\Gamma(\delta)} \frac{\partial^{k}}{\partial \delta^{k}}\left[\frac{\Gamma(\delta)}{\alpha^{\delta-1}}\right]$.

\section{Appendix C. A linear combination for the quantity $f(x ; \delta, \alpha, \xi) F(x ; \delta, \alpha, \xi)^{v}$}

First, we derive a power series expansion for $F(x ; \delta, \alpha, \boldsymbol{\xi})^{v}$. From equation (2.1), we have

$F(x ; \boldsymbol{\delta}, \alpha, \boldsymbol{\xi})^{v}=\left(\frac{[\alpha H(x ; \boldsymbol{\xi})]^{\delta}}{\Gamma(\boldsymbol{\delta})}\right)^{v}\left(\sum_{m=0}^{\infty} \frac{(-\alpha)^{m}}{(\boldsymbol{\delta}+m) m !} H(x ; \boldsymbol{\xi})^{m}\right)^{v}=\left(\frac{[\alpha H(x ; \boldsymbol{\xi})]^{\delta}}{\Gamma(\boldsymbol{\delta})}\right)^{v}\left(\sum_{m=0}^{\infty} w_{m} H(x ; \boldsymbol{\xi})^{m}\right)^{v}$,

where $w_{m}=(-\alpha)^{m} /[(\delta+m) m !]$. We assume that $v$ is a positive integer, and then the Eq. A.1 implies that $F(x ; \boldsymbol{\delta}, \alpha, \boldsymbol{\xi})^{v}=\left(\frac{[\alpha H(x ; \boldsymbol{\xi})]^{\delta}}{\Gamma(\boldsymbol{\delta})}\right)^{v} \sum_{m=0}^{\infty} w_{m, v} H(x ; \boldsymbol{\xi})^{m}=\sum_{m=0}^{\infty} w_{m, v}\left(\frac{\alpha^{\delta}}{\Gamma(\boldsymbol{\delta})}\right)^{v} H(x ; \boldsymbol{\xi})^{m+v \delta}=\sum_{m=0}^{\infty} s_{m, v} H(x ; \boldsymbol{\xi})^{m+v \delta}$,

where $s_{m, v}=w_{m, v}\left(\frac{\alpha^{\delta}}{\Gamma(\delta)}\right)^{v}$ and the coefficients $w_{m, v}$ for $m=1,2, \ldots$ are obtained from the recurrence relation in Eq. A.1. Combining this result and the expansion C.1], we have

$$
f(x ; \boldsymbol{\delta}, \alpha, \boldsymbol{\xi}) F(x ; \boldsymbol{\delta}, \alpha, \boldsymbol{\xi})^{v} \sum_{m=0}^{\infty} s_{m, v} H(x ; \boldsymbol{\xi})^{m+v \delta}=\frac{\alpha^{\delta-1}}{\Gamma(\boldsymbol{\delta})} g(x ; \alpha, \boldsymbol{\xi}) \sum_{m=0}^{\infty} s_{m, v} H(x ; \boldsymbol{\xi})^{m+\delta(v+1)-1},
$$

where the last equation holds because of (1.4). 


\section{References}

[1] M. Bebbington, C.D. Lai and R. Zitikis, "A flexible Weibull extension," Reliability Engineering \& System Safety, 92, 719 - 726 (2007).

[2] A.L. Bowley, Elements of Statistics, King (1920).

[3] J.M.F. Carrasco, E.M.M. Ortega and G.M. Cordeiro, "A generalized modified Weibull distribution for lifetime modeling," Computational Statistics and Data Analysis, 53, 450 - 462 (2008).

[4] Z. Chen, "A new two-parameter lifetime distribution with bathtub shape or increasing failure rate function," Statistics and Probability Letters, 49, 155 -161 (2000).

[5] T.M. Cover and J.A. Thomas, Elements of Information Theory, John Wiley \& Sons (2006).

[6] B. Gompertz, "On the nature of the function expressive of the law of human mortality and on the new model of determining the value of life contingencies," Philosophical Transactions of the Royal Society of London, 115, 513 - 585 (1825).

[7] I.S. Gradshteyn and I.M. Ryzhik, Table of Integrals, Series, and Products, Academic Press (2007).

[8] M. Gurvich, A. DiBenedetto and S. Ranade, "A new statistical distribution for characterizing the random strength of brittle materials," Journal of Materials Science, 32, 2559-2564 (1997).

[9] N.L. Johnson, S. Kotz and N. Balakrishnan, Continuous Univariate Distributions, New York: John Wiley \& Sons (1994).

[10] J.A. Kies, "The strength of glass," Washington D.C., Naval Research Lab, Report 5093 (1958).

[11] C.D. Lai, M. Xie and D.N.P. Murthy, "A modified Weibull distribution," IEEE Transactions on Reliability, 52, 33 - 37 (2003).

[12] J.J.A. Moors, "A quantile alternative for kurtosis," Journal of the Royal Statistical Society (Series D), 37, 25-32 (1988).

[13] D.N.P. Murthy, M. Xie and R. Jiang, Weibull Models, John Wiley \& Sons (2004).

[14] S. Nadarajah and S. Kotz, "On some recent modifications of Weibull distribution," IEEE Transactions on Reliability, 54, 561 - 562 (2005).

[15] M. Nikulin and F. Haghighi, "A chi-squared test for the generalized power Weibull family for the headand-neck cancer censored data," Journal of Mathematical Sciences, 133, 1333 - 1341 (2006).

[16] H. Pham, "A vtub-shaped hazard rate function with applications to system safety," International Journal of Reliability and Applications, 3, 1 -16 (2002).

[17] K.K. Phani, "A new modified Weibull distribution function," Communications of the American Ceramic Society 70, 182 - 184 (1987).

[18] J.W.S. Rayleigh, "On the resultant of a large number of vibrations of the same pitch and of arbitrary phase," Philosophical Magazine Series 5, 10, 73-78 (1880).

[19] R.M. Smith and L.J. Bain, "An exponential power life testing distribution," Communications in Statistics, Theory and Methods, 4, 469 - 481 (1975).

[20] G. Steinbrecher and W.T. Shaw, "Quantile mechanics," European Journal of Applied Mathematics, 19, 87-112 (2008).

[21] J.S. White, “The moments of log-Weibull order statistics," Technometrics, 11, 373 - 386 (1969).

[22] M.M. Xie and D. Lai, "Reliability analysis using additive Weibull model with bathtub-shaped failure rate function," Reliability Engineering \& System Safety, 52, 87 - 93 (1995).

[23] M. Xie, Y. Tang, T.N. Goh, "A modified Weibull extension with bathtub-shaped failure rate function," Reliability Engineering \& System Safety, 76, 279 - 285 (2002).

[24] K. Zografos and N. Balakrishnan, "On families of beta and generalized gamma-generated distributions and associated inference,” Statistical Methodology, 6, 344-362 (2009). 